\title{
Sensitivity Analysis of Linear Programming and Quadratic Programming Algorithms for Control Allocation
}

\author{
Susan A. Frost ${ }^{1}$ \\ NASA Ames Research Center, Moffett Field, CA 94035 \\ Marc Bodson ${ }^{2}$ \\ University of Utah, Salt Lake City, UT 84112 \\ and \\ Diana M. Acosta ${ }^{3}$ \\ NASA Ames Research Center, Moffett Field, CA 94035
}

\begin{abstract}
The Next Generation (NextGen) transport aircraft configurations being investigated as part of the NASA Aeronautics Subsonic Fixed Wing Project have more control surfaces, or control effectors, than existing transport aircraft configurations. Conventional flight control is achieved through two symmetric elevators, two antisymmetric ailerons, and a rudder. The five effectors, reduced to three command variables, produce moments along the three main axes of the aircraft and enable the pilot to control the attitude and flight path of the aircraft. The NextGen aircraft will have additional redundant control effectors to control the three moments, creating a situation where the aircraft is over-actuated and where a simple relationship does not exist anymore between the required effector deflections and the desired moments. NextGen flight controllers will incorporate control allocation algorithms to determine the optimal effector commands and attain the desired moments, taking into account the effector limits. Approaches to solving the problem using linear programming and quadratic programming algorithms have been proposed and tested. It is of great interest to understand their relative advantages and disadvantages and how design parameters may affect their properties. In this paper, we investigate the sensitivity of the effector commands with respect to the desired moments and show on some examples that the solutions provided using the $l_{2}$ norm of quadratic programming are less sensitive than those using the $l_{1}$ norm of linear programming.
\end{abstract}

${ }^{1}$ Research Engineer, Intelligent Systems Division, M/S 269-1, and AIAA Member.

${ }^{2}$ Professor, Electrical \& Computer Engineering Department, 50 S Central Campus Dr Rm 3280, and AIAA Senior Member.

${ }^{3}$ Research Engineer, Intelligent Systems Division, M/S 269-1, and AIAA Member. 


\section{Introduction}

Concern for the environmental and operational impacts of civil aviation is prompting significant changes in civil aviation. The NASA Aeronautics Subsonic Fixed Wing Project, together with industry, universities, and other government organizations, is researching advanced technologies and exploring novel civil transport configurations to achieve the desired reduction in noise, emissions, fuel consumption, and field length for Next Generation (NextGen) aircraft ${ }^{1-2}$. Potential NextGen transport airplanes include the Cruise Efficient Short Take-Off and Landing (CESTOL) and the Hybrid Wing-Body (HWB) aircraft. See Ref. 2 for detailed information on these new NextGen architectures and their control challenges.

The advanced configurations and associated technologies for NextGen aircraft elevate the complexity of the vehicle and its operation. Flight control will be particularly challenging, in part because of the added demands on the control allocation schemes. Unlike conventional control allocation schemes that control three body-fixed rates primarily with three control variables, NextGen control allocation schemes will need to control the three body-fixed rates with a variety of redundant and multi-objective control surfaces ${ }^{3}$. The control allocation of over-actuated aircraft has been formulated as a constrained optimization problem by some researchers ${ }^{4}$. Ultimately, a real-time solution to the control allocation problem is desirable to enable the system to run on an aircraft during flight. This has prompted the search for numerical optimization methods that have good convergence properties and acceptable computational requirements.

Several methods to solve the control allocation problem have been evaluated, including direct allocation, linear programming, quadratic programming, weighted pseudo-inverse, cascaded generalized inverse, and a mixed optimization approach. ${ }^{4,5}$ Control allocation research has also extended the control solution to include coupling or interaction effects between control effectors, creating a nonlinear optimization problem which can often be transformed into a linear problem $^{6,7}$. While the interaction effects will be important to study for control allocation in NextGen aircraft, we focus in this paper on solutions that assume a linear relationship between the effectors and the moments.

Two optimization methods that have been shown to be effective and implementable in realtime are the linear programming (LP) and the quadratic programming (QP) methods ${ }^{4-5}$. Previous studies, however, have not addressed the issue of the sensitivity of the solution to the data. In particular, the sensitivity of the solution with respect to the desired moments determines how rapidly the control effectors have to move if the moments are to match the desired moments. The sensitivity of two control allocation algorithms, one using an LP method and the other using a QP method, are evaluated in this paper by examining their application in two examples of overactuated aircraft.

\section{Control Allocation in Flight Control Systems}

Control allocation is the stage in the stability and control augmentation process that is responsible for achieving a commanded moment vector by allocating commands to individual control effectors. NextGen flight control algorithms will control $q$ body-fixed angular accelerations or moments of the aircraft with $n$ control effectors, where $n>q$.

In its simplest practical form, the control allocation scheme will consider the rate and magnitude limits of the control effectors. The control allocation problem can be posed in state space form as follows: find the $m \times 1$ control vector, $u_{c m d}$, such that 


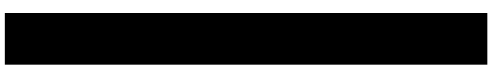

subject to

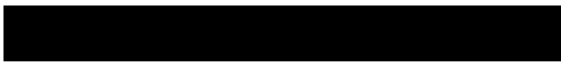

where $B$ is the $q \times m$ linear control effectiveness matrix, is the $q \times 1$ commanded angular acceleration vector, $m_{c m d}$ is the $q \times 1$ commanded moment vector, $u_{\min }$ and $u_{\max }$ are the vectors of the control effectors' minimum and maximum magnitude limits, respectively, and is the vector of control effectors' maximum rate limits. In this paper, we set aside the rate limits and focus on the moments that are achievable, without considering the previous deflections that may have been applied.

The control allocation algorithm should optimally allocate the available control effectors to solve Eq. (1), while limiting unachievable commands. The attainable moment set for a given operating point of an aircraft is the set of acceleration moments that can be achieved by the control effectors without violating their saturation limits. If the solution to Eq. (2) is not unique, additional objectives, such as control minimization, can be added to the optimization problem to yield a preferred solution.

\section{A. Mixed Optimization Approach to Control Allocation}

The mixed optimization approach, as proposed by Bodson ${ }^{5}$, combines the error minimization and the control minimization into one problem by minimizing the cost function:

$$
J=\left\|B u_{c m d}-\dot{x}_{c m d}\right\|_{1}+\varepsilon\left\|u_{c m d}-u_{p r e f}\right\|_{1}
$$

subject to

$$
u_{\min } \leq u_{c m d} \leq u_{\max }
$$

where $u_{\min }$ and $u_{\max }$ are the vectors of the control effectors' minimum and maximum magnitude limits, $0 \leq \varepsilon \leq 1$ is a control minimization weight, and $u_{\text {pref }}$ is a preferred control vector. If the value of the parameter $\varepsilon$ is small, priority will be given to error minimization over control minimization, as desired. Note that the optimization criterion is based on the $l_{1}$ norm, which is the sum of the absolute values of the component of the vector.

The mixed optimization approach expands upon work performed by Buffington ${ }^{9}$, who suggested that control allocation could be solved using a sequential optimization approach in which the error minimization problem is first solved followed by solution of the control minimization problem. The specific choice of $l_{1}$ norm enabled Buffington to solve the control allocation problem with linear programming techniques ${ }^{9}$, providing guaranteed convergence to a solution in an acceptable period of time.

The mixed optimization approach, as implemented by Bodson ${ }^{5}$, combined the two optimization steps into one, and was shown to be the most effective LP optimization method in an evaluation of different approaches used in simulations of a C-17 aircraft model and a tailless aircraft model. The algorithm was based on the revised simplex method ${ }^{10}$ with additional refinements as described in detail in Ref. 5, which enabled computations to be performed 
extremely fast, and well within the capabilities of modern computers. This paper uses the same code for the LP optimization method in the comparison studies that follow.

\section{B. Active Set Methods for Solving Quadratic Control Allocation}

Quadratic programming or Least-Squares (LS) methods make use of the $l_{2}$ norm and have

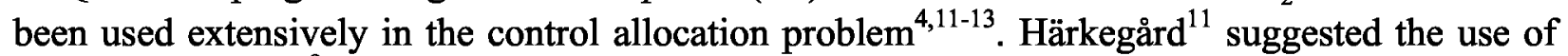
active set methods $^{8}$ for quadratic solutions to the control allocation problem. Active set methods can be shown to find an optimal solution in a finite number of steps ${ }^{9}$. A comparison of constrained quadratic programming techniques for control allocation found that the weighted least-squares (WLS) form of the active set method performed favorably for control allocation of over-actuated aircraft that resemble NextGen aircraft configurations ${ }^{12}$. The active set algorithm is to quadratic programming what the simplex algorithm is to linear programming. The steps of the algorithm are very comparable, and the solutions can be computed very fast with both methods.

The general problem formulation for the quadratic solution to the mixed optimization control allocation problem is to minimize the cost function:

$$
J=\gamma\left\|W_{e}\left(B u_{c m d}-\dot{x}_{c m d}\right)\right\|_{2}^{2}+\left\|W_{c}\left(u_{c m d}-u_{p r e f}\right)\right\|_{2}^{2}
$$

subject to

$$
u_{\min } \leq u_{c m d} \leq u_{\max }
$$

where $u_{\min }$ and $u_{\max }$ are the vectors of the control effectors' minimum and maximum magnitude limits, $W_{e}$ is the $(q \times q)$ acceleration error weighting matrix, $W_{c}$ is the $(n \times n)$ control error weighting matrix, $\gamma$ is a control error weight scalar, and $u_{\text {pref }}$ is a preferred control vector. Note that the optimization criterion in Eq. (5) is essentially identical to the criterion in Eq. (3), except for the choice of $l_{2}$ norm instead of $l_{1}$ norm. This leaves open the question of whether the choice of norm makes any difference in the solutions obtained. The point of this paper is to show that it does. The WLS algorithm ${ }^{11,13}$ was used as the QP method for analysis in this paper. See Ref. 13 for the source code and documentation on the WLS algorithm.

\section{Computational Results}

Computations were performed on a model of a C17 transport aircraft with 16 control effectors and a model based on Lockheed Martin's ICE (innovative control effectors) tailless aircraft ${ }^{15}$ with 11 control effectors, using the data of Ref. 5. The parameters used in the simulations were as follows. In the LP control allocation scheme, $\varepsilon=1 e-6$. In the QP control allocation scheme, $\gamma=1 e+6$.

Figures 1-4 compare the results of computations for the $\mathrm{C} 17$ with a pure yaw acceleration command given as the desired moment, e.g., the pitch and roll acceleration components were identically zero. The simulation input had the yaw moment start at 0 and step up to 8.72 degrees $/ \mathrm{sec}^{2}$ which is less than the maximum attainable yaw moment of $8.7232 \mathrm{degrees} / \mathrm{sec}^{2}$ for the $\mathrm{C} 17$ model. There were 100 points in the interval. Figure 5 shows the commanded effector deflections for the $\mathrm{C} 17$ model using the QP algorithm with the input being a pure roll acceleration starting at 0 and stepping up to 61.07 degrees $/ \mathrm{sec}^{2}$, which is less than the attainable 
roll moment of 61.0741 degrees $/ \mathrm{sec}^{2}$. There were 100 points in the simulation interval and the desired pitch and yaw accelerations were identically zero. Figures 6-9 compare the results of computations of the ICE with a pure roll acceleration command given as the desired moment, e.g., the pitch and yaw acceleration components were identically zero. The computation had the roll moment start at 0 and step up to 212.0 degrees $/ \mathrm{sec}^{2}$ which is less than the maximum attainable roll moment of 212.062 degrees/ $\mathrm{sec}^{2}$ for the ICE model. There were 100 points in the simulation interval.

Comparison of fig. 1 with fig. 2 reveals that the allocation of the control effectors is more distributed in the QP solution. Also, the changes in the control effector deflections are generally more progressive in the QP solution. In fig. 1, the commanded deflections from the LP control allocation algorithm sometimes proceed in a non-monotonic manner. This feature is not necessarily problematic, but it is not desirable either.

Figure 3 shows that the errors between the accelerations commanded by the flight controller and the accelerations achieved by using the commanded deflections were larger for the QP method. Interestingly, a difference between the $l_{1}$ and $l_{2}$ solutions is that, for $\varepsilon$ small, the $l_{1}$ solution does not constitute a trade-off between performance (error) and cost (control), but rather produces results similar to a two-step optimization procedure where error is minimized first, and control is minimized within this solution as a secondary objective. This feature may be part of the reason for the increased sensitivity. Nevertheless the QP error is small and could be made smaller by changing $\gamma$.

An interesting observation in the plots is that both methods show increased sensitivity when the commanded accelerations approach the achievable acceleration limit. Figures 1 and 5 provide good examples of this "terminal" sensitivity for the LP and the QP methods, respectively. The terminal sensitivity is a problematic feature for both methods that will be further investigated, together with methods to reduce it.

In order to quantify the sensitivity, a sensitivity measure was computed as the $l_{2}$ norm of the difference between the commanded deflections for a given yaw moment and the commanded deflections of the same yaw moment with a small constant added to it. The constant was 0.01 times the maximum attainable yaw moment for the aircraft at that operating point. Figure 4 shows the sensitivity measure for the LP and the QP methods when only a pure yaw moment is used for the commanded acceleration. As expected, the sensitivity measure is greater for the LP algorithm. As mentioned earlier, both algorithms seemed to have their sensitivities increase once one of the control effectors became saturated. Figures 6-9 tell a similar story as fig. 1-4, they also demonstrate that similar results are obtained for another aircraft with a different configuration of control effectors. 


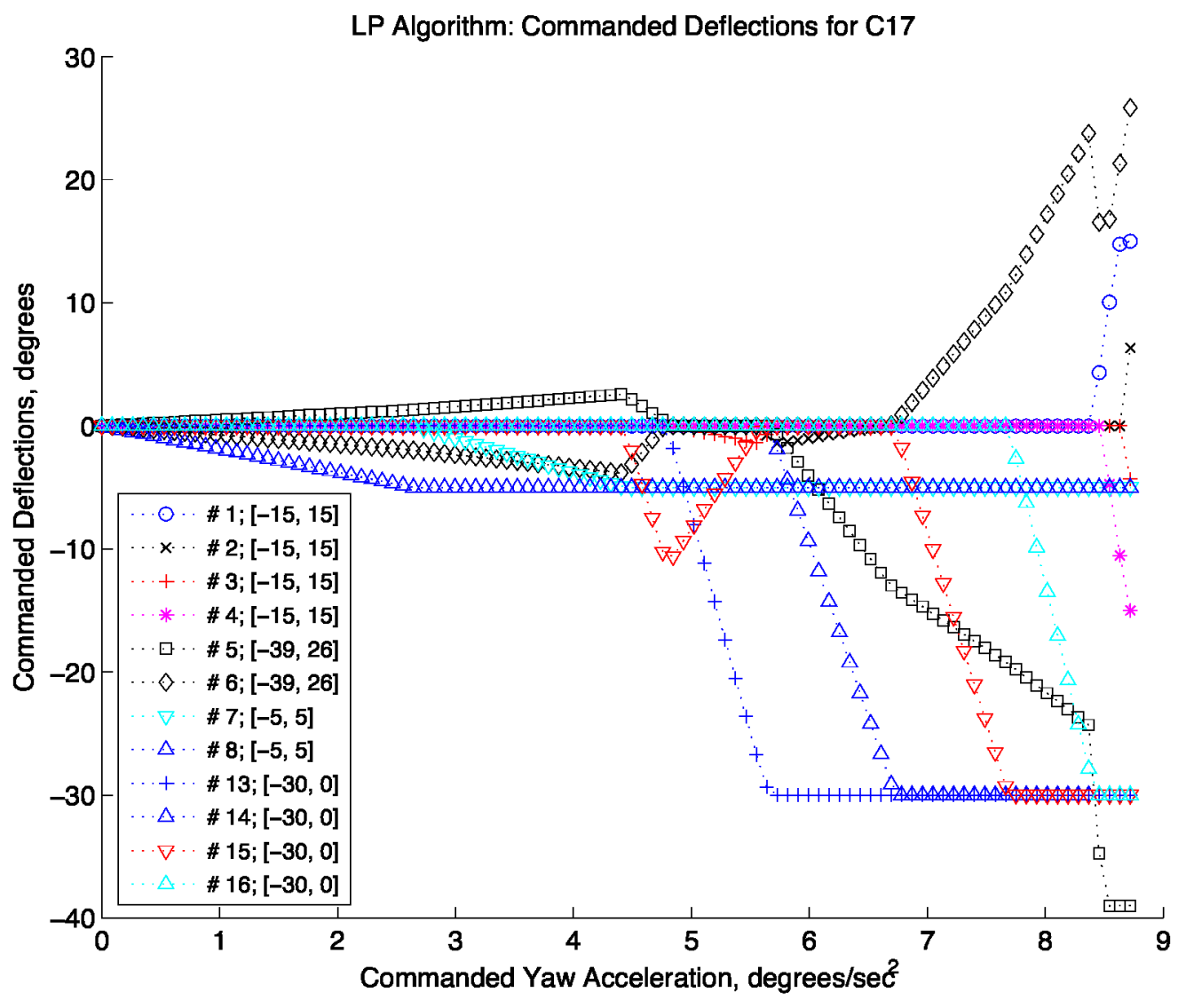

Figure 1. Commanded deflections from LP control allocation algorithm for $\mathrm{C17}$ with pure commanded yaw acceleration. 


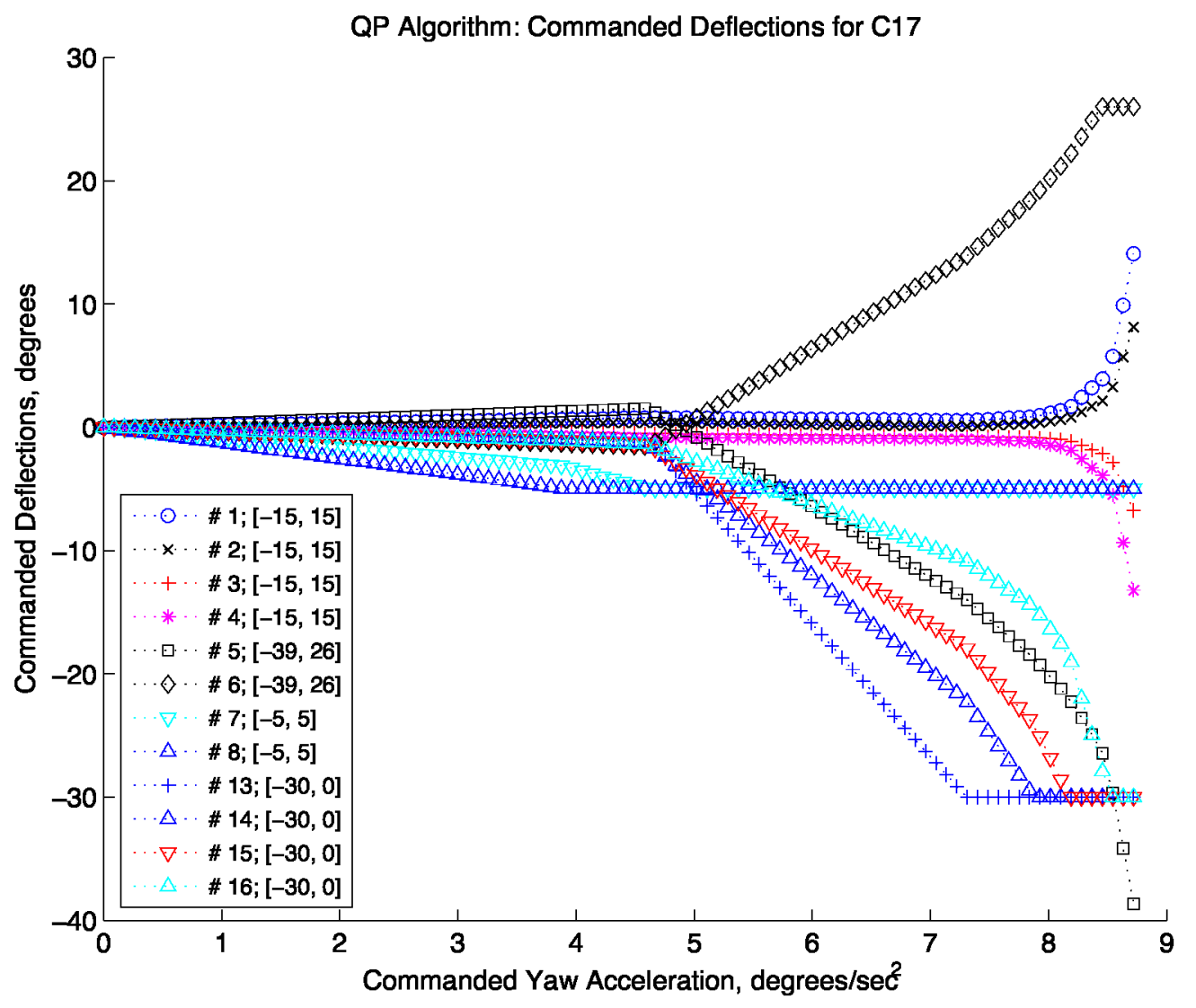

Figure 2. Commanded deflections from QP control allocation algorithm for C17 with pure commanded yaw acceleration. 

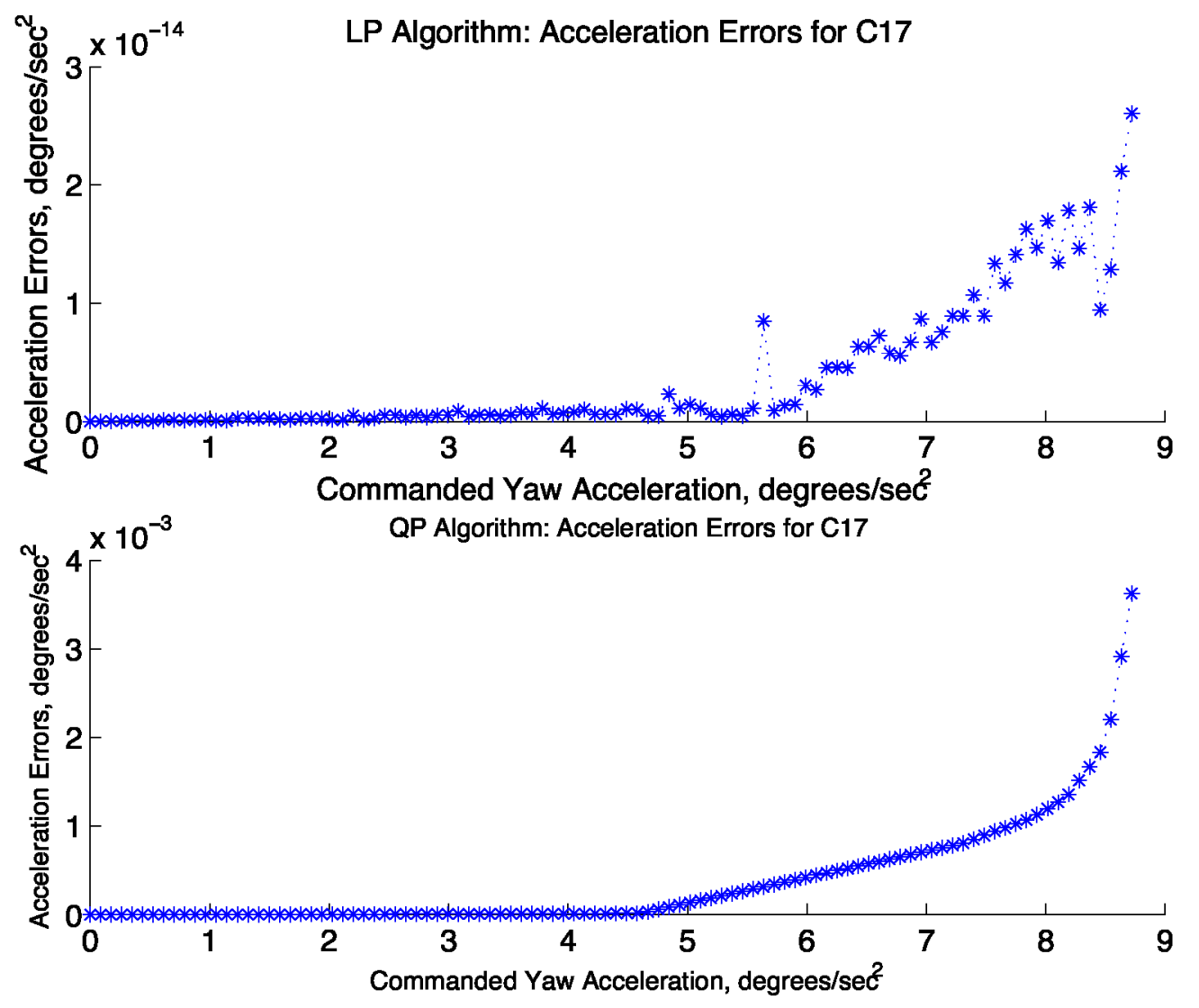

Figure 3. a) Acceleration errors for LP control allocation algorithms for C17 with pure commanded yaw acceleration, b) Acceleration errors for QP algorithm. 

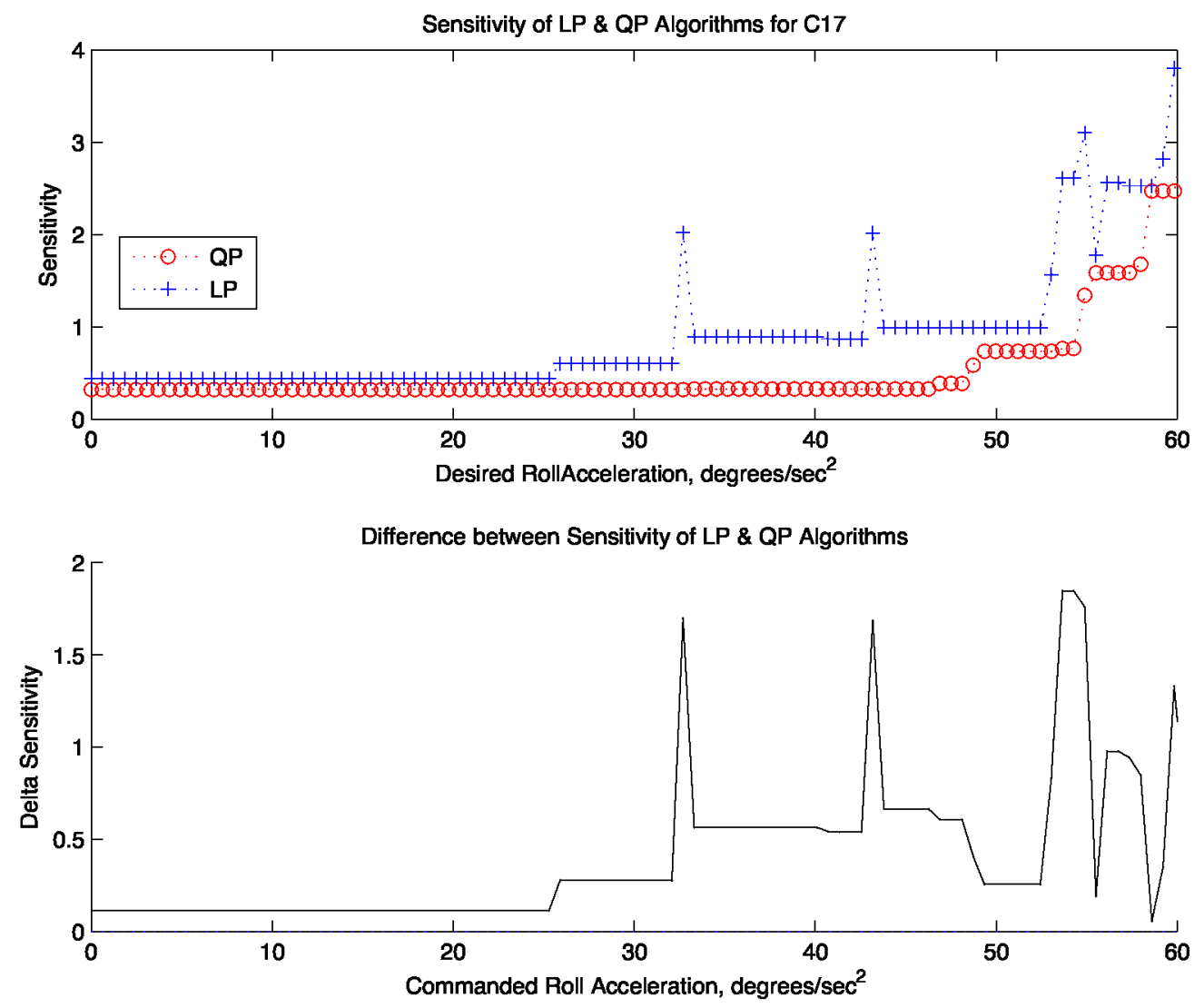

Figure 4. a) Sensitivity of LP \& QP control allocation algorithms for C17 with pure commanded yaw acceleration, b) difference between LP \& QP sensitivities. 


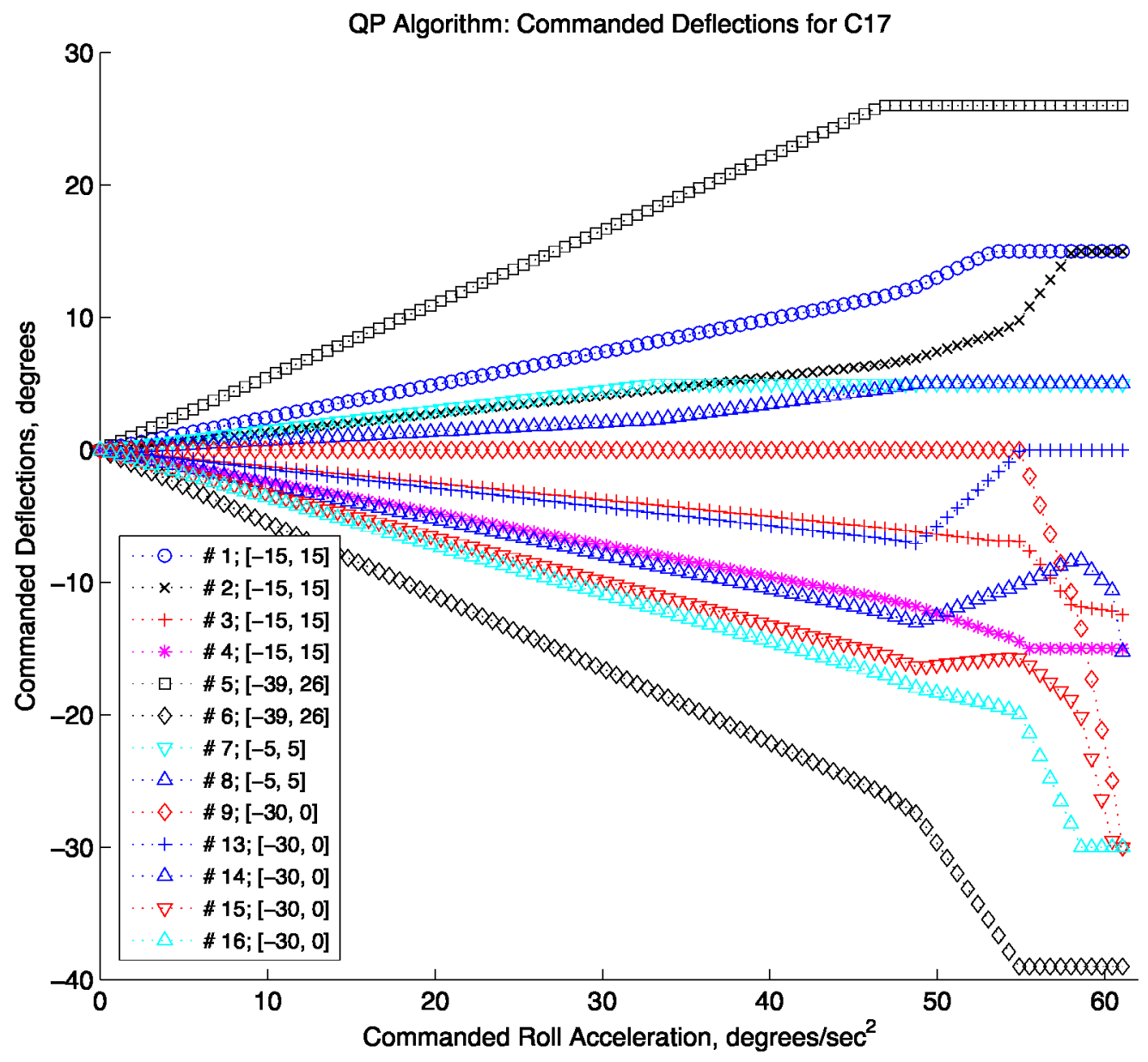

Figure 5. Commanded deflections from QP control allocation algorithm for C17 with pure commanded roll acceleration. 


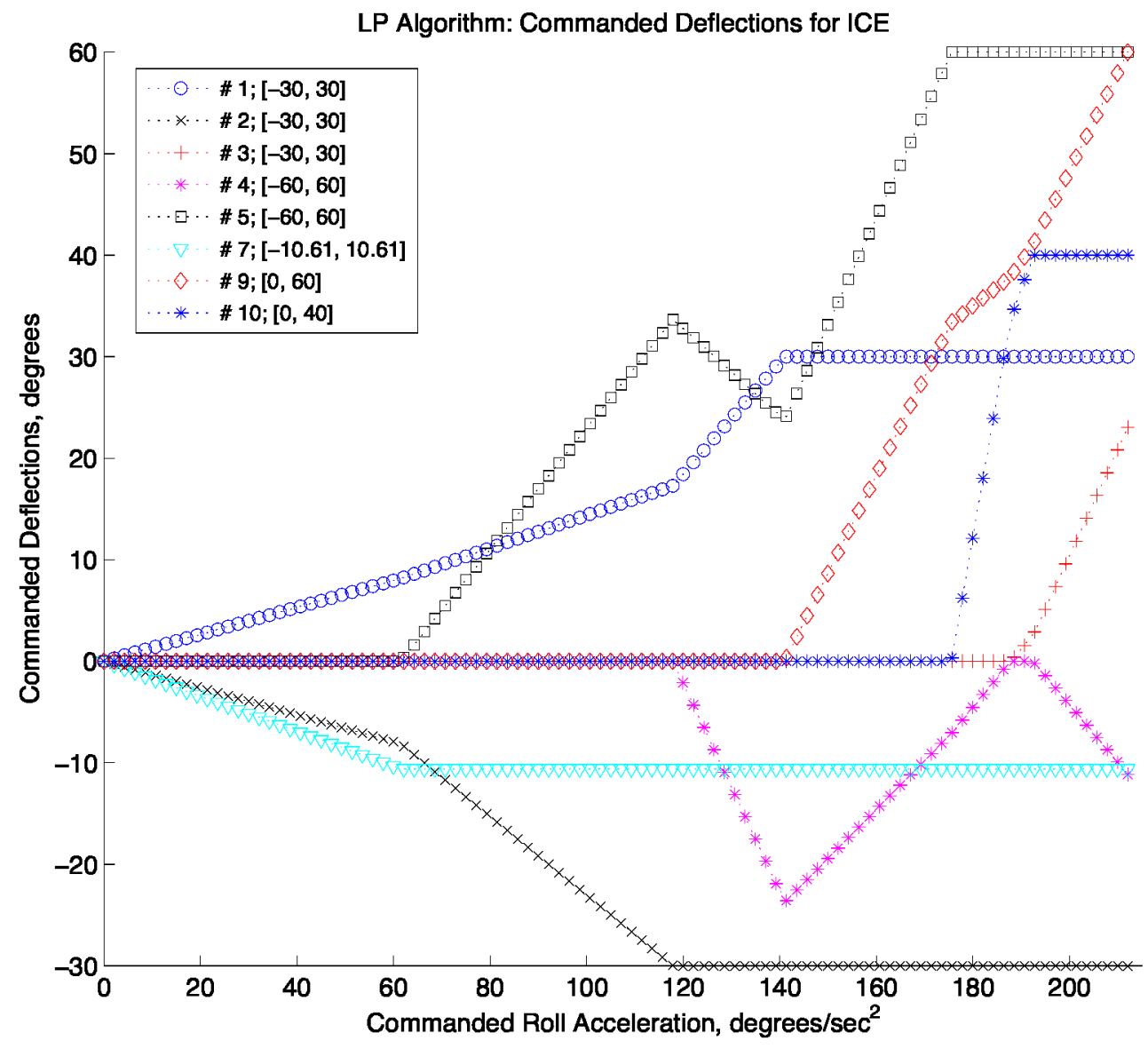

Figure 6. Commanded deflections from LP control allocation algorithm for ICE with pure commanded roll acceleration. 


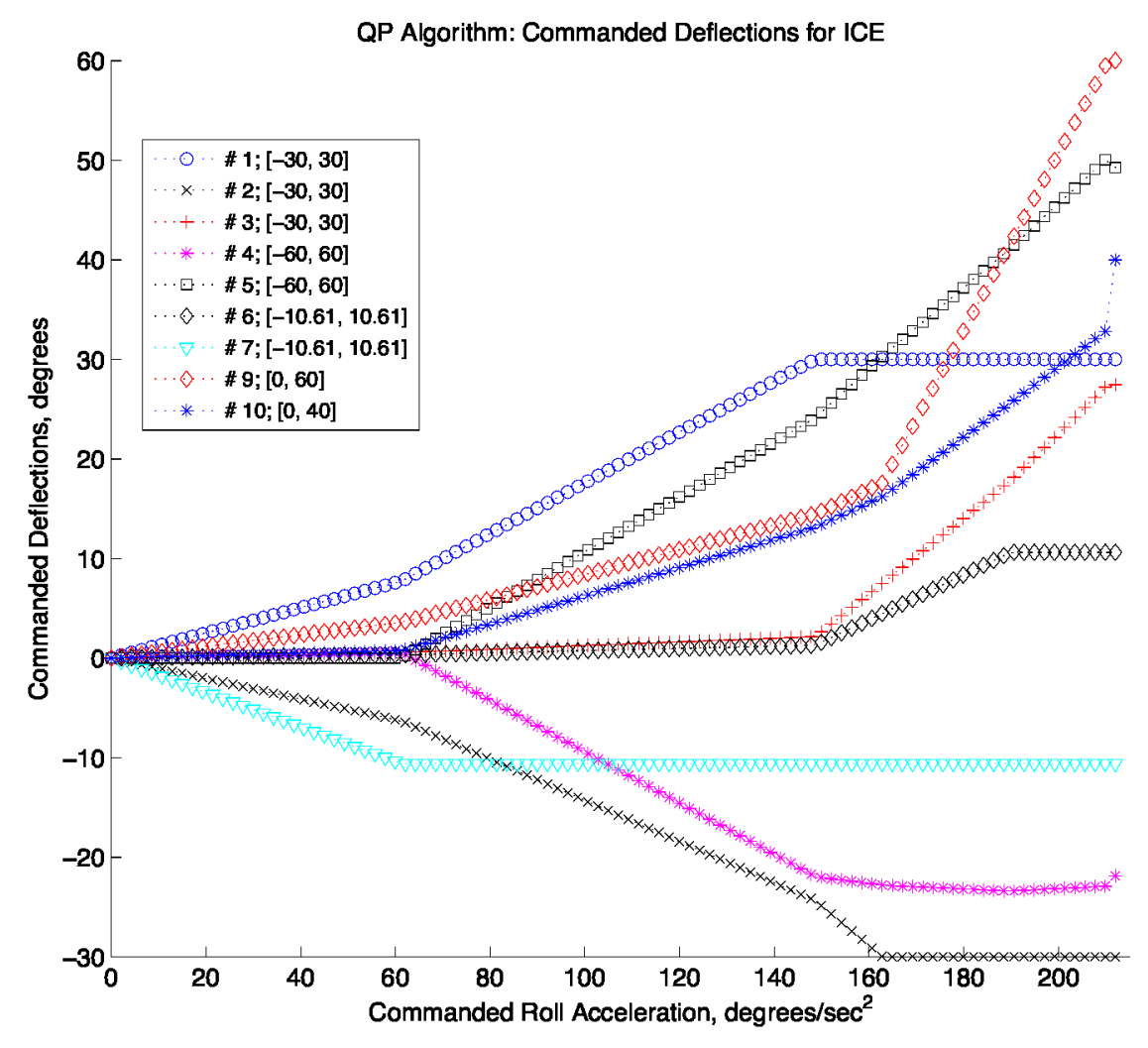

Figure 7. Commanded deflections from QP control allocation algorithm for ICE with pure commanded roll acceleration. 

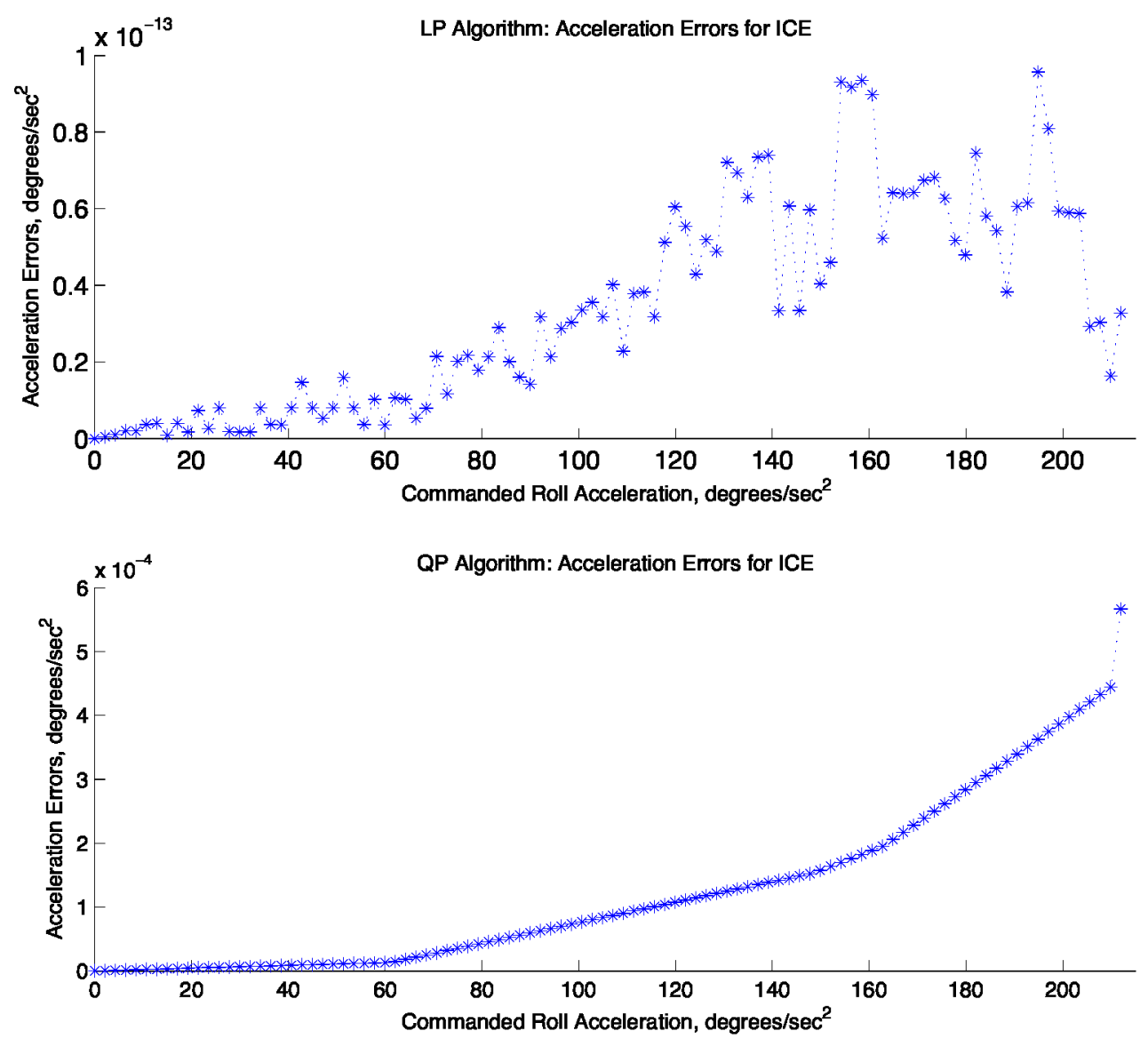

Figure 8. a) Acceleration errors for LP control allocation algorithms for ICE with pure commanded roll acceleration, b) acceleration errors for QP algorithm. 

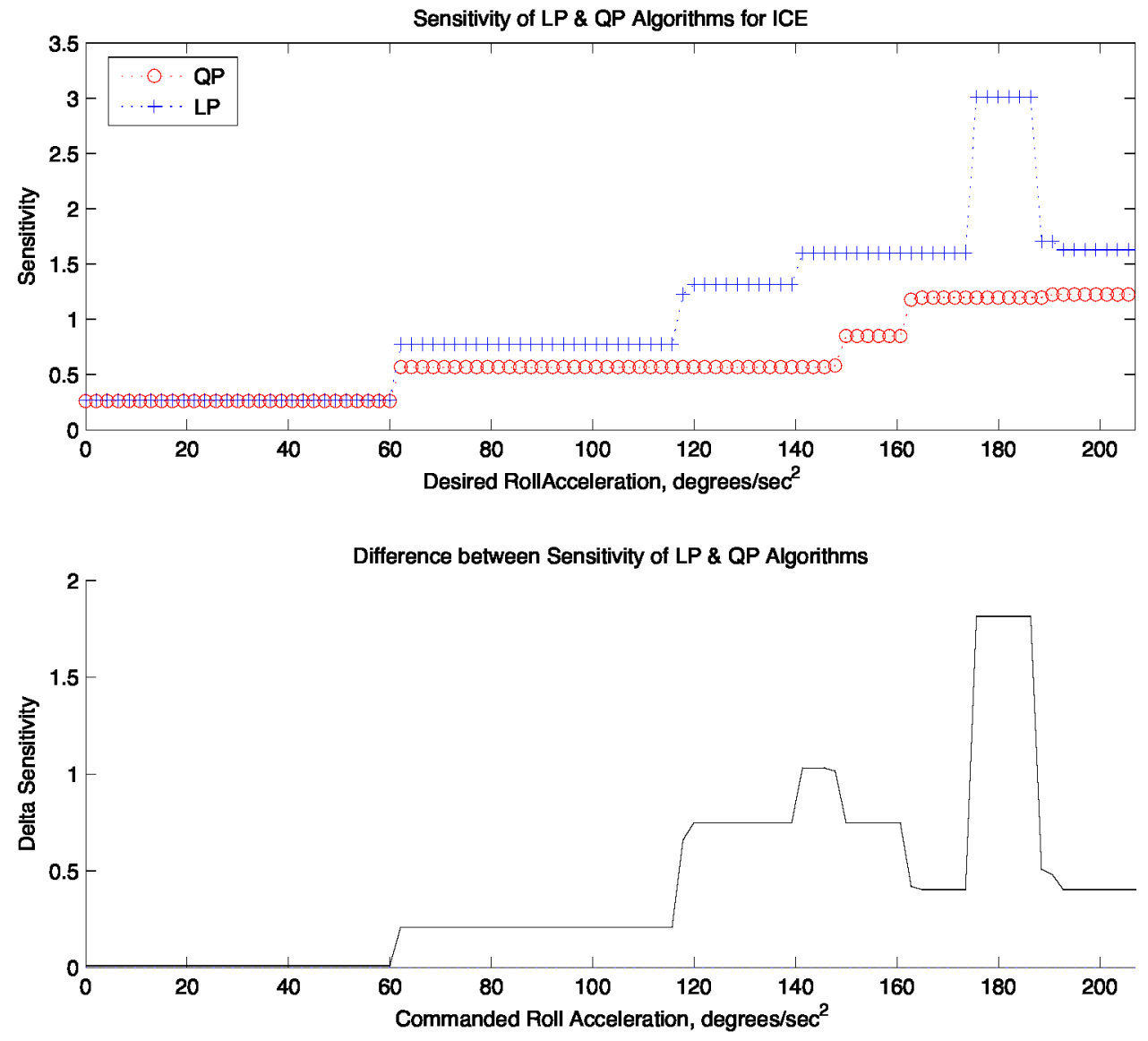

Figure 9. a) Sensitivity of LP \& QP control allocation algorithms for ICE with pure commanded roll acceleration, b) difference between LP \& QP sensitivities.

\section{Conclusion}

Comparisons were made between control allocation schemes using an LP algorithm and a QP algorithm for minimizing an $l_{1}$ and an $l_{2}$ cost function, respectively. It was observed that the allocation of the control effectors appears to be more distributed in the QP control allocation computation. Also, the changes in the control effector deflections are generally smoother in the QP computations. The final paper will present sensitivity results based on statistical sampling of vectors from inside and outside the attainable moment set for the application. The preliminary results suggest that designers, especially those working on NextGen aircraft, would be wise to examine the sensitivity of the chosen control allocation algorithm for their application. Additional research will also be directed towards understanding the sensitivity at the boundary of the attainable set for both the LP and the QP algorithms. 


\section{References}

1 "Fundamental Aeronautics Program Subsonic Fixed Wing Project Reference Document," National Aeronautics and Space Administration, May 2006, URL: http://www.aeronautics.nasa.gov/programs_fap.htm

${ }^{2}$ Acosta, D.M., KrishnaKumar, K., and Frost, S. A.. "Towards Intelligent Control for Next Generation Aircraft," Proceedings International Powered Lift Conference, 2008 Royal Aeronautical Society, London, England, July 2008.

${ }^{3}$ Cameron, D., Princen, N., "Control Allocation Challenges and Requirements for the Blended Wing Body," AIAA Guidance, Navigation and Control Conference and Exhibit, AIAA, Denver, Colorado, August 2000, AIAA-2000-4539.

${ }^{4}$ Page, A. B., Steinberg, M. L., "High-Fidelity Simulation Testing of Control Allocation Methods," AIAA Guidance, Navigation and Control Conference and Exhibit, AIAA, Monterey, California, August 2002, AIAA-2002-4547.

5 Bodson, M., "Evaluation of Optimization Methods for Control Allocation," AIAA Guidance, Navigation, and Control Conference and Exhibit, August 2001, Montreal, Canada, AIAA-2001-4223.

${ }^{6}$ Davidson J.B., Lallman, F. J., and Bundick, W. T., "Real-Time Adaptive Control Allocation Applied to a High Performance Aircraft," $5^{\text {th }}$ SIAM Conference on Control \& Its Applications, 2001.

${ }^{7}$ Oppenheimer, M. W. and Doman, D. B., "A Method for Including Control Effector Interactions in the Control Allocation Problem," report AFRL-VA-WP-TP-2007-309, Air Force Research Laboratory, Wright-Patterson AFB, OH 45433-7542, 2007.

${ }^{8}$ Nocedal, J. and Wright, S. J., Numerical Optimization, Springer, New York, 1999.

${ }^{9}$ Buffington, J., "Modular Control Law Design for the Innovative Control Effectors (ICE) Tailless Fighter Aircraft Configuration 101-3," report AFRL-VA-WP-TR-1999-3057, Air Force Research Laboratory, Wright-Patterson AFB OH 45433$7542,1999$.

${ }^{10}$ Luenberger, D., Introduction to Linear and Nonlinear Programming, Addison-Wesley, Reading, MA 1984, pp. 80.

${ }^{11}$ Härkegård, O., "Efficient Active Set Algorithms for Solving Constrained Least Squares Problems in Aircraft Control Allocation," $41^{\text {st }}$ IEEE Conference on Decision and Control, Vol. 2, pp. 1295-1300.

${ }^{12}$ Peterson, J. A. M. and Bodson, M., "Constrained Quadratic Programming Techniques for Control Allocation," IEEE Transactions on Control Systems Technology," Vol. 14, No. 1, January 2006, pp. 91-98.

${ }^{13}$ Enns, D., "Control Allocation Approaches," AIAA Guidance, Navigation and Control Conference and Exhibit, AIAA, Boston, MA, August 1998, AIAA-1998-4109.

14"Quadratic Programming Control Allocation Toolbox (QCAT)," Matlab® Central, August 2008, URL: http://www.mathworks.com/matlabcentral/fileexchange/loadFile.do?objectId=4609\&objectType=file

${ }^{15}$ Peterson, J. A. M. and Bodson, M., "Command Limiting in Reconfigurable Flight Control," AIAA J. of Guidance, Control, and Dynamics," Vol. 21, No. 4, 1998, pp. 639-646. 